\title{
Analysis of the Result of Korean Medicine Treatment for Female Subfertility Using Herbal Medicine, Acupuncture and Moxibustion Treatment
}

\author{
Jae-Cheol Jeong ${ }^{1}$, Min-Sun Choi ${ }^{1}$, Sang-Ho Yoon ${ }^{2}$, Dong-Il Kim ${ }^{1}$ \\ ${ }^{1}$ Department of Obstetrics \& Gynecology, College of Korean Medicine, Dongguk University \\ ${ }^{2}$ Department of Obstetrics \& Gynecology, College of Medicine, Dongguk University
}

\begin{abstract}
Objectives: To report on the result of infertility treatment with Korean medicine in clinical settings.
Methods: This study was single arm, retrospective observational study. Analysis of the medical records on thirty four infertile women who were treated by acupuncture, moxibustion, herbal medicine(Jokyungjongoktang-gagambang, Sutaehwan-gagambang). Pregnancy rate(PR) and Live birth rate(LBR) were analyzed as the primary outcomes. Changes of $\mathrm{AMH}, \mathrm{FSH}, \mathrm{LH}, \mathrm{E}_{2}, \mathrm{P}_{4}$, endometrial thickness, fat mass, and BMI were also analyzed as the secondary outcomes.

Results: A total of 34 patients were treated in the traditional Korean medical clinic. Nine patients were dropped, and 25 subjects completed the final examination at the end of this treatment schedule. Five of 25 subjects conceived during treatment, reaching 20\% PR and LBR was also 20\%. Except for FSH level, the results of the remaining outcome measures, including LFT, showed no significant changes. No serious adverse event has been reported. Conclusion: Although no significant changes in bio-markers were observed, complex treatment using Korean medicine has shown effectiveness on women's infertility. In addition, the safety of the treatment has been verified.
\end{abstract}
Key Words : infertility, subfertility, pregnancy rate, acupuncture, herbal medicine, traditional Korean medicine

\section{Introduction}

South Korea is known as having one of the lowest birth rates in the world, and infertility has been thought to be an important factor causing the low birth rate. As this has been become a social issue, the government has implemented policies to resolve it. The government reinforced economic support for infertile couples in taking assistant reproductive technology(ART) as a main program. This program has helped many infertile couples become pregnant; however, the clinical pregnancy rate(PR) by this project only increased the success rate to around $30 \%$ per cycle. In addition, the ART program was associated with significant stress for infertile couples [1].

Traditional Korean medicine has a long history in treatment of infertility. The effect of Korean medicine has been verified not only in the traditional medical books [2-4], but also in recent studies [5,6]. Based on this supporting evidence and the medical environment, more than $70 \%$ of infertile couples

\footnotetext{
- Received : 25 December 2014

- Revised : 17 June 2015

- Accepted : 17 June 2015

- Correspondence to: Dong-II Kim

Department of Obstetrics \& Gynecology, College of Korean Medicine, Dongguk University

814 Siksa-dong, Ilsandong-gu, Goyang-City, Gyeonggi-province, South Korea

Tel : +82-31-961-9062, Cell phone : +82-10-4518-7820, E-mail : obgykdi@dongguk.ac.kr
} 
visited traditional Korean medicine clinics [1]. Thus, we conducted retrospective study from our clinic data in order to overcome the limits of ART to help people suffering from infertility.

Herbal medicine Jokyungjongoktang-gagambang (JKJOT-GGB) and Sutaehwan-gagambang(STH-GGB), acupuncture, and moxibustion treatment were used in our clinic. JKJOT has been widely used in improving female infertility, especially that induced by stress [7]. Some studies have reported on the effect of JKJOT associated with immunological mechanism on stress, hormone changes, and ovulation [8-11]. STH is an herbal medicine used for prevention of miscarriage and some studies have reported that STH was effective for treatment of recurrent miscarriage, threatened abortion. The decoction worked as a positive influence on the maternal immune system for carrying on pregnancy [12-15].

Various studies on the relations between acupuncture and treatment of infertility have been reported. According to in vivo and clinical studies, acupuncture may regulate the function of the hypothalamic-pituitary-ovarian axis and change the concentration of neuropeptides such as $\beta$-endorphin, and also affect uterine blood flow and uterine motility [14]. Results of a meta-analysis study showed that acupuncture improved clinical PR and LBR among women undergoing IVF [5].

These evidence-based treatments have been well utilized in traditional Korean clinics. We provided infertile patients with complex and effective treatments.

\section{Materials and Methods}

\section{Study design and participants}

This study was conducted from the medical records of the infertile female patients who had visited at Dongguk University Ilsan Korean medicine
hospital(DUIH) between July 2011 and June 2012.

The inclusion criteria were as follows: women unsuccessful at conceiving for more than one year, even after many attempts to become pregnant; women diagnosed with unexplained infertility or ovulation related infertility from an infertility clinic; women who had intercourse with reasonable frequency at least twice or more per week, except during the menstrual period; women who approved of sharing the results.

The exclusion criteria were as follows: women who did not maintain the treatment with acupuncture and herbs for the duration of six months; taking medication for infertility, like hormonal drugs; diagnosed with polycystic ovarian syndrome(PCOS); male infertility; taking psychiatric medications.

Patients' visiting schedule was set by their own menstrual cycle day (MCD). Each patient visited the hospital four times per menstrual cycle, at MCD3, 8, 14, and 21. Assessments were performed and treatments were administered during six cycles, thus, each patient made a total of 24 hospital visits.

Each patient visited the hospital on MCD3 for the screening. Patients received acupuncture with moxibustion treatment and JKJOT-GGB was given on sight (MCD3). Blood chemistry test was performed for observation of changes of $\mathrm{FSH}, \mathrm{LH}$, estrogen, AMH, and LFT. BMI, fat mass, and endometrial thickness were also measured on the first visit.

Acupuncture and moxibustion treatments were administered on the first, second, and third visit (MCD 3, 8, and 14). On the third visit(MCD14), patients received STH-GGB and sonogram was performed for measurement of endometrial thickness (SA8000, SoNOAce PICO, Samsung medison, Korea). On the fourth visit(MCD21), blood chemistry test was performed for measurement of the progesterone level.

All the patients agreed with providing their medical information for this study. And information 
of patients was used solely for the purposes of this study. This protocol was approved by DUIH IRB (2014-03)

\section{Treatments}

\section{1) Herbal medicine}

Both herbal medicines, JKJOT-GGB and STH -GGB, were purchased from Dongguk University Ilsan Korean medicine hospital. Participants took JKJOT-GGB for 10 days and STH-GGB for 15 days, twice per day. Each extract was prepared by decocting the dried prescription with water. The contents of the decoction are as follows.

JKJOT-GGB consists of 15 herbs; roots of Rehmannia glutinosa(GAERTNER) LIBOSCH (Scrophulariaceae) (12g), rhizomes of Cyperus rotundus L.(Cyperaceae) (12g), roots of Angelica gigas NAKAI(Umbelliferae) (8g), fruits of Evodia rutaecarpa BENTH(Rutaceae) $(6 \mathrm{~g})$, rhizomes of Cnidium officinale MAKINO(Umbelliferae) (6g), roots of Paeonia lactiflora PALL.(Ranunculaceae) (8g), Sclerotia of Poriacocos(SCHW.) WOLF (Polyporaceae) (8g), fruit skins of Citrus unshiu MARKOVICH(Rutaceae) (6g), tubers of Corydalis turtschaninovii BESS(Papaveraceae) (6g), cortexes of Paeonia suffruticosa ANDREWS(Ranunculaceae) (4g), dried rhizomes of Zingiber officinale ROSC. (Zingiberaceae) (4g), cortexes of Cinnamomum cassia PRESL(Lauraceae) (4g), leaves of Artemisia vulgaris L.(Compositae) (6g), velvet antler of Cervusnippon TEMMINCK.(Cervidae) (8g), rhizomes of Zingiber officinale ROSC.(Zingiberaceae) (3g).

STH-GGB consists of six herbs; fruits of Cuscuta chinensis LAM.(Convolvulaceae) (12g), rhizomes of Dioscorea japonica THUNB.(Dioscoreaceae) (24g), roots of Dipsacusasper WALL. (Dipsacaceae) (8g), roots of Astragalus membranaceus BUNGE (Leguminosae) (8g), roots of Angelica gigas NAKAI(Umbelliferae) (4g), roots of Panax ginseng C.A. MEY.(Araliaceae) (3g).
2) Acupuncture and moxibustion

Two doctors of Korean medicine administered all treatments. Both were qualified by the Korean ministry of health and welfare; one has had more than 20 years of clinical experience and the other has had more than two years of clinical experience. All patients were treated in the supine position and received acupuncture and moxibustion treatments three times during each cycle.

For the acupuncture treatment, 10 points were used, based on the traditional and contemporary literature reviews and consensus of doctors of Korean medicine who are experts in women's health(Table 1). Sterile disposable $0.25 \times 40 \mathrm{~mm}$ needles(Dongbang Acupuncture Inc., Korea) were used, and the retention time was $20 \pm 3 \mathrm{~min}$ per treatment. Depths of needling varied from 5 to $30 \mathrm{~mm}$ depending on the acupoints. The doctor manipulated the needles until the participant felt deqi sensation and electro-acupuncture stimulation was set to a continuous wave at $2 \mathrm{~Hz}$, which was applied on two sets, SP6 at one end and SP9 at the other as one set and M-CA-18 and N-CA-4 as another set. During acupuncture treatment, TDP(Teding diancibo $\mathrm{Pu}$ ) was applied on the lower abdomen and participants were allowed to speak freely with doctors regarding their symptoms and conditions.

For the moxibustion treatment, Charcoal moxa (Dongbang Acupuncture Inc., Korea) was applied on

Table 1. Acupuncture Points of the Project

\begin{tabular}{|c|c|c|}
\hline $\begin{array}{c}\text { international number } \\
(\mathrm{WHO})\end{array}$ & detail & Additional stimulation \\
\hline GV20 & unilateral & \\
\hline $\mathrm{CV} 4$ & unilateral & \\
\hline SP6 & bilateral & electro acupuncture \\
\hline SP9 & bilateral & (SP6-SP9, bilateral) \\
\hline M-CA-18*(Extra point) & bilateral & electro acupuncture \\
\hline N-CA-4 ${ }^{\dagger}$ (Extra point) & bilateral & (Zigong-Tituo, bilateral) \\
\hline
\end{tabular}

("JaGung: Its literal meaning is "uterus". On the lower abdomen, 4 cun $(\fallingdotseq 6-8 \mathrm{~cm})$ below the center of the umbilicus and 3 cun $(\fallingdotseq$ $4.5-6 \mathrm{~cm})$ lateral toCV3. †JeTak: Its literal meaning is "lift and support". 4cun $(\fallingdotseq 6-8 \mathrm{~cm})$ lateral to the anterior midline and 2cun $(\fallingdotseq$ $3-4 \mathrm{~cm})$ superior to the upper border of the pubic symphysis.) 
the CV4 acupoint for 15 mins per treatment, immediately after acupuncture treatment.

\section{Outcomes}

PR within the six menstrual cycle period and LBR were set as the primary outcome measures. The secondary outcome measure was the changes of hormone levels in FSH, LH, estrogen, and AMH. Hormone levels were checked during the first and sixth menstrual cycle periods. BMI, fat mass, and endometrial thickness were also measured during the same menstrual cycle periods.

\section{Statistical method}

SAS statistical package version 9.1 was used. Paired t-test was used for continuous variables. Differences of $P<0.05$ were considered significant.

\section{Results}

\section{Participant flow}

Thirty four women were treated for infertility in our clinic. Nine women were excluded due to PCOS, spouse factor, drop out of the treatment schedule, resulting in 25 participants were analyzed. Data on blood chemistry test and LFT of five pregnant women were not acquired, thus, the analysis was performed using data from 20 women who failed to

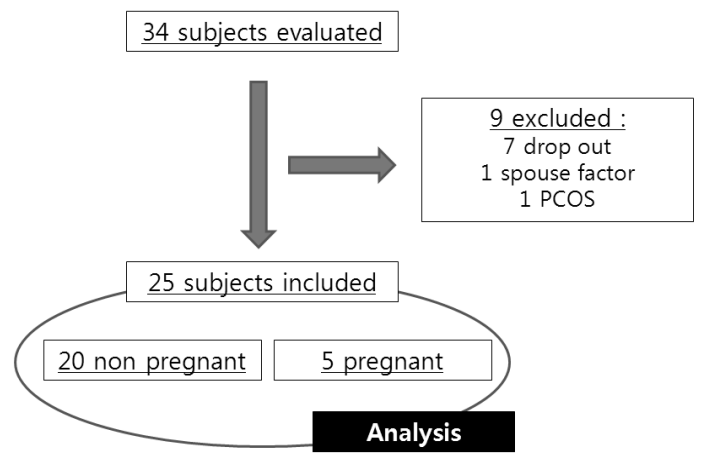

Fig. 1. Flow chart of Patients Disposition
conceive(Figure 1).

\section{Baseline data}

Baseline characteristics are shown in Table 2 . Most patients, except for one patient, had undergone other types of treatment for infertility.

Table 2. General and Gynecological Characteristics of Participants

\begin{tabular}{lrr}
\hline & $\mathrm{n}$ & \multicolumn{1}{c}{$\mathrm{M}(\mathrm{SD})$} \\
\hline General characteristics & & \\
Age, yr & 25 & $33(2.18)$ \\
Height, cm & 25 & $162.12(4.26)$ \\
Body weight, kg & 25 & $56.44(13.37)$ \\
Body fat, \% & 25 & $30.2(6.6)$ \\
BMI, kg/m ${ }^{2}$ & 25 & $21.42(4.39)$ \\
\hline Gynecological characteristics & & \\
Duration of trying pregnancy, number. & 25 & $41.36(24.98)$ \\
Duration of treatment, month & 25 & $17.93(16.86)$ \\
Age of menarche, year & 25 & $13.28(1.14)$ \\
Amount of menstruation, pad & 25 & $17.88(6.48)$ \\
Duration of menstruation, day & 25 & $4.96(1.4)$ \\
Menstrual cycle, day & 25 & $29.36(2.38)$ \\
\hline
\end{tabular}

\section{Pregnancy rate}

Five of 25 patients succeeded in conceiving and the PR per treatment of six cycles was $20 \%$ $(5 / 25 * 100)$.

Two women conceived during cycle 1 and another three women conceived during the following cycles: 2, 3, and 6 (Table 3).

Table 3. PR and LBR

\begin{tabular}{cccc}
\hline $\begin{array}{c}\text { Pregnancy Time } \\
\text { (menstrual cycle) }\end{array}$ & Number & PR(\%) & LBR(\%) \\
\hline Cycle1 & 2 & & \\
Cycle2 & 1 & & \\
Cycle3 & 1 & & \\
Cycle6 & 1 & & \\
within 6 cycles & $5 / 25$ & 20 & 20 \\
\hline
\end{tabular}

\section{Live birth rate}

All of five pregnancies succeeded to result in 
birth and the LBR was $20 \%(5 / 25 * 100)$. (Table 3$)$. There were three cases of spontaneous labor, and two cases of cesarean section.

5. Changes of hormone level, BMI, body fat mass and endometrial thickness

Changes in hormone levels between cycles 1 and 6 were not statistically significant, except FSH. No significant differences were observed in AMH ( $P=$ 0.9477), LH $(P=0.1247)$, Estrogen $(P=0.7775)$, and Progesterone $(P=0.5876)$, however, significant difference was observed in FSH $(P=0.0366)$.

BMI and body fat mass during cycles 1 and 6 showed no significant difference. Endometrial thickness measures on MCD 3 and 14 also showed no significant difference after treatment. The results are shown in Table 4.

\section{Adverse events}

There were four mild adverse events; one complaint of heartburn, two complaints of constipation after taking herbal medicine, and one complaint of nausea after acupuncture treatment. However, no severe adverse event was observed. The changes of LFT were not statistically significant (Table 4).

\section{Discussion}

Through this project, five participants conceived within six menstrual cycles. Therefore, the PR was $20 \%$ in six cycles of this project. In comparison with the result of the national supporting program for infertile couples in Korea(31.2\% in 2007, 31.9\% in 2008 , and $30.2 \%$ in 2009), the PR of our program was lower than the PR of past programs [1]. However, in our program, there was a limitation in controlling the number of times the subject had intercourse twice or more per week, referring to another study. For example, one study reported that patients with infertility had significantly low scores in the desire and arousal domains and low frequency of intercourse and masturbation; and another study reported that depression, erectile dysfunction, and problems in sexual relationships were prevalent among male partners of infertile couples [16,17].

It is important to increase the PR, however, ultimately, increasing LBR is more important for infertile couples. In addition, in this project, as

Table 4. Changes of Secondary Outcomes

\begin{tabular}{|c|c|c|c|c|}
\hline & $\begin{array}{l}\text { Cycle 1, } \\
\text { M(SD) }\end{array}$ & $\begin{array}{l}\text { Cycle 2, } \\
\text { M(SD) }\end{array}$ & $\mathrm{n}$ & P-value \\
\hline \multicolumn{5}{|l|}{ Changes of hormone level } \\
\hline $\mathrm{AMH}(\mathrm{ng} / \mathrm{ml})$ & $3.65(3.16)$ & $3.61(2.54)$ & 20 & 0.9477 \\
\hline FSH(mIU/ml) & $7.49(2.7)$ & $8.76(4.34)$ & 20 & $0.0366^{*}$ \\
\hline $\mathrm{LH}(\mathrm{mIU} / \mathrm{ml})$ & $4.9(2.23)$ & $7.16(6.17)$ & 20 & 0.1247 \\
\hline Estradiol(pg/ml) & $43.15(19.49)$ & $42.07(19.21)$ & 20 & 0.7775 \\
\hline Progesterone $(\mathrm{ng} / \mathrm{ml})$ & $10.87(7.05)$ & $9.84(6.08)$ & 20 & 0.5876 \\
\hline \multicolumn{5}{|c|}{ Changes of endometrial thickness, fat mass and BMI } \\
\hline Endometrial thickness, MCD3 (mm) & $3.68(1.61)$ & $2.87(1.39)$ & 20 & 0.0535 \\
\hline Endometrial thickness, MCD14 (mm) & $7.93(2.42)$ & $8.07(1.54)$ & 20 & 0.7851 \\
\hline Fat mass $(\%)$ & $29.16(6.34)$ & $29.31(7.32)$ & 20 & 0.9094 \\
\hline $\mathrm{BMI}\left(\mathrm{kg} / \mathrm{m}^{2}\right)$ & $21.49(4.88)$ & $21.71(5.08)$ & 20 & 0.0794 \\
\hline \multicolumn{5}{|l|}{ Changes of LFT } \\
\hline AST(IU/L) & $19.45(10.28)$ & $20.7(9.56)$ & 20 & 0.5407 \\
\hline ALT(IU/L) & $13.55(13.16)$ & $19.65(17.29)$ & 20 & 0.0743 \\
\hline ALP(IU/L) & $50.05(12.28)$ & $49.25(13.7)$ & 20 & 0.3979 \\
\hline
\end{tabular}


STH-GGB was prescribed for implantation and prevention of miscarriage, comparison of $\mathrm{BR}$ would be more reasonable than PR. In this project, there was no occurrence of miscarriage, therefore, LBR was the same as PR, that is, $20 \%$ in six cycles of pregnancy. Compared to the result of ART, $21.3 \%$ in $2006,22.8 \%$ in 2007 , this result demonstrated the potential of Korean medicine in prevention of miscarriage [1].

To determine influential factors for possible pregnancy in the future, hormone test, BMI, body fat mass, and endometrial thickness measurement were performed for participants who failed to conceive. FSH level only showed a significant increase, but the rest showed no significant change. This result indicated that the treatments might not lead to decrease of ovarian reserve and increase of BMI.

On the other hand, considering the increase of FSH and decreasing tendency(not statistical) of $\mathrm{AMH}$, it appeared that traditional Korean treatments could not prevent or slow the process of aging of ovarian function [18]. Thus, it is necessary to consider the age factor of women and the duration of treatment at the beginning of treatment planning.

During the six-month treatment period, there was no occurrence of hepatotoxicity by LFT or severe adverse events and only four mild adverse events were observed. Although the duration of treatment was extensive, the treatment was safe for the participants.

Due to small sample size, lack of comparable objective measurement, measurement errors due to differences of menstrual cycle, and exclusion of successfully conceived women's post examination data, there are limitations to interpreting the findings. Regardless of such limitations, through this project, we achieved significant results, with a PR of $20 \%$, and a LBR of $20 \%$ (no miscarriage). We also proved the safety of herbal medical treatment via hepatotoxicity, maintaining ovarian reserve and BMI.

\section{Conclusion}

Although no significant changes were observed in endometrial thickness, hormone levels, except FSH, the complex treatments using Korean medicine, JKJOT-GGB, STH-GGB, acupuncture and moxibustion, have shown effectiveness in management of women's infertility, especially PR and LBR. In addition, the safety of the treatment has been verified. Further clinical research is needed in order to evaluate the efficacy more objectively.

\section{References}

1. Ministry of health and welfare of Korea, Medical college of Seoul national university. Analysis and evaluation of the national supporting program for infertility couples in 2008. 2009.

2. Heo Jun. Trans. Committee of translating Dong-ui-bo-gam. Dong-ui-bo-gam. Seoul:Bubin publishers company. 1999.

3. Jang GB. Gyeong-ak-jeon-seo, Bu-in-gyu. Trans. Lee TG. Seoul:Bubin publishers company. 1999.

4. Jin JM. Bu-in-dae-jeon-yang-bang. Trans. Kim DI, et al. Seoul:Gwangsam publishers company. 2003.

5. Zheng $\mathrm{CH}$, Huang GY, Zhang MM, Wang W. Effects of acupuncture on pregnancy rates in women undergoing in vitro fertilization: a systematic review and meta-analysis. Fertil Steril. 2012;97:599-611.

6. Reid K, Stuart K. Efficacty of traditional Chinese herbal medicine in the management of female infertility: A systematic review. Complement Ther Med. 2011;19:319-331.

7. Hwang DY. Bangyakhappyeon. Seoul:Yeonglim publishers company. 2002.

8. Lee HS, Cho KH, Kim TK, Kim CH, Ahn SJ, Lee EH, et al. Cho-kyung-jong-ok-tang, a traditional Korean herbal formula induces type2 
shift in murine natural killer cell cytokine production. Jour Ethnopharmaco. 2011;134:281-287.

9. Min GH, Kim JY, Kim SB, Cho HB. Effects of Chokyungjongoktang on immune system by immobilization stress in $\mathrm{C} 57 \mathrm{BL} / 6 \mathrm{~J}$ mice. The journal of oriental obstetrics and gynecology. 2004; 17:46-60.

10. Yang JM, Seo IB, Lee DN, Kim HJ. Effects of Jokyeongjonggk-Tang on the maturation of follicles and NGF expression in rats with estradiol valerate-induced polycystic ovaries. The journal of oriental obstetrics and gynecology. 2011;24:52-67.

11. Bae JK, Kim GW. Effect of Jokyungjongoktang on the fluctuation of gonadotropin and sex hormone concentration in serum of women. The journal of dongguk oriental medicine. 1992;1: $15-54$.

12. Fan L. The clinical observation of added Shoutai pill on treating habitual abortion. Journal of new Chinese medicine. 2011;43: 81-83.

13. Lai M, You Z, Ma H, Lei L, Lu F, He D, et al. Effects of Shoutai pills on expression of Th1/Th2 cytokine in maternal-fetal interface and pregnancy outcome. Zhongguo Zhong Yao Za Zhi. 2010;35:3065-3068.

14. NG EH, So WS, Gao J, Wong YY, Ho PC. The role of acupuncture in the management of subfertility. Fertil Steril. 2008;09:1-13.

15. Choi JK, Sin YW, Jung JH, Yu DR. Effect of Sutaehwan on immune response in pregnant BALB/C mice. The journal of oriental obstetrics and gynecology. 2003;16:87-97.

16. Millheiser LS, Helmer AE, Quintero RB, Westphal LM, Milki AA, Lathi RB. Is infertility a risk factor for female sexual dysfunction? A case-control study. Fertil Steril. 2010;94:20222025.

17. Shindel AW, Nelson CJ, Naughton CK, Ohebshalom M, Mulhall JP. Sexual function and quality of life in the male partner of infertile couples: prevalence and correlates of dysfunction. J Urol. 2008; 179:1056-1059.

18. van Rooij IA, Broekmans FJ, Scheffer GJ, Looman CW, Habbema JD, de Jong FH, et al. Serum antimullerian hormone levels best reflect the reproductive decline with age in normal women with proven fertility: a longitudinal study. Fertil Steril. 2005;83:979-987. 